\title{
Expanding the clinical spectrum of 3-phosphoglycerate dehydrogenase deficiency
}

\author{
L. Tabatabaie • L. W. J. Klomp • M. E. Rubio-Gozalbo • \\ L. J. M. Spaapen • A. A. M. Haagen • L. Dorland • \\ T. J. de Koning
}

Received: 19 March 2010/Revised: 3 November 2010 /Accepted: 8 November 2010 /Published online: 27 November 2010

(C) The Author(s) 2010. This article is published with open access at Springerlink.com

\begin{abstract}
Phosphoglycerate dehydrogenase (3-PGDH) deficiency is considered to be a rare cause of congenital microcephaly, infantile onset of intractable seizures and severe psychomotor retardation. Here, we report for the first time a very mild form of genetically confirmed 3-PGDH deficiency in two siblings with juvenile onset of absence seizures and mild developmental delay. Amino acid analysis showed serine values in CSF and plasma identical to what is observed in the severe infantile form. Both patients responded favourably to relatively low dosages of serine supplementation with cessation of seizures, normalisation of their EEG abnormalities and improvement of well-being and behaviour. These cases illustrate that 3-PGDH deficiency can present with mild
\end{abstract}

Communicated by: Jaak Jaeken

Competing interests: None declared.

L. Tabatabaie $\cdot$ L. W. J. Klomp

Department of Metabolic and Endocrine Diseases, University

Medical Center Utrecht,

Utrecht, The Netherlands

\section{E. Rubio-Gozalbo}

Department of Pediatrics, University Medical Centre Maastricht, Utrecht, The Netherlands

M. E. Rubio-Gozalbo $\cdot$ L. J. M. Spaapen · L. Dorland Department of Biochemical Genetics, University Medical Centre Maastricht,

Maastricht, The Netherlands

A. A. M. Haagen

Department of Pediatrics, VieCuri Medical Centre Venlo, Venlo, The Netherlands

L. Tabatabaie $\cdot$ T. J. de Koning $(\bowtie)$

Department of Metabolic Diseases, University Medical Center Utrecht,

KC 03.063.0, PO Box 85090, 3508 AB Utrecht, The Netherlands

e-mail: t.dekoning@umcutrecht.nl symptoms and should be considered as a treatable disorder in the differential diagnosis of mild developmental delay and seizures. Synopsis: we present a novel mild phenotype in patients with 3-PGDH deficiency.

\section{Introduction}

3-Phosphoglycerate dehydrogenase deficiency (OMIM $\# 601815)$ is an autosomal recessive disorder caused by a defect in the synthesis of the amino acid L-serine. The disorder was first reported by Jaeken and colleagues in 1996 in two patients with congenital microcephaly, intractable seizures and severe psychomotor retardation (Jaeken et al. 1996). After this initial report, additional patients were documented in the literature and from these reports a rather homogeneous clinical phenotype evolved (de Koning et al. 1998; de Koning 2006; Hausler et al. 2001; Jaeken et al. 1996; Pind et al. 2002; Pineda et al. 2000). All patients except one, presented with congenital microcephaly indicative of the fact that in the majority of patients the symptoms already occurred before birth. Affected infants developed intractable seizures within weeks to months after birth and showed little to no psychomotor development. Finally, a severe spastic quadriplegia becomes evident during the first years of life. In addition, the patients were irritable, hypertonic, had feeding difficulties and appeared to be "unhappy" infants. EEG recordings showed several seizure types, including hypsarithmia and multifocal epileptic activity (de Koning et al. 2002). Cranial MRI scans showed a striking absence of white matter volume and hypomyelination (de Koning et al. 2000). In the patients with this severe infantile phenotype the response to oral treatment with L-serine sometimes combined with glycine was favourable with respect to their well-being and 
seizures. In some patients the seizures completely resolved while in others seizures reappeared after some period, albeit in a lower frequency. The results of amino acid treatment on the progression of psychomotor development, however were very disappointing, with little to none progression of psychomotor development in symptomatic patients during long term follow-up (de Koning 2006).

In this paper we present a family with a hitherto unreported very mild phenotype of 3-PGDH deficiency, expanding the clinical phenotype to that of juvenile onset of seizures with mild psychomotor retardation.

\section{Case reports}

Two children, a boy and a girl, were born to healthy nonconsanguineous Dutch parents. In the boy the pregnancy was complicated by intrauterine growth retardation. At birth, his height was $48 \mathrm{~cm}$ (3rd centile), weight 2600 grams (3rd centile) and head circumference $33 \mathrm{~cm}$ ( 3 rd centile). He was slow in acquiring early developmental milestones (pulls to stand at 10 months, first words at 18 months, walking at 24 months) and after three years of age developmental delay became evident. At the age of nine years he presented with absence seizures (complex typical absences) and his EEG recording showed bilateral synchronous spike-wave complexes, which were enhanced after hyperventilation with typical $3 \mathrm{~Hz}$ spike wave complexes. His absence seizures were successfully treated with ethosuximide. An attempt of ethosuximide withdrawal at the age of 15 years was unsuccessful. He visits a secondary school for children with learning difficulties and is actively involved in sports.

At the time of diagnosis he was 16 years old and demonstrated hyperactive behaviour and mild developmental delay with an IQ of 49. His head circumference was $55 \mathrm{~cm}$ (10th centile) whereas his height and weight were both at the 75th centile. His neurological exam was unremarkable except for a subtle bilateral ankle clonus. In addition, he had generalised hyperlaxity of large and small joints and striae rubra on his abdomen and upper legs. His cranial MRI revealed no abnormalities. Laboratory investigations were performed in this family because the parents requested genetic counselling for familial seizures. Extensive investigations revealed no abnormalities, except that his plasma serine was at the lower end of the normal range (63 $\mu \mathrm{mol} / \mathrm{L}$, reference range $70-174)$ with a normal value for glycine $(252 \mu \mathrm{mol} / \mathrm{L}$, reference 147-321). However, his cerebrospinal fluid (CSF) serine concentration was severely reduced with a value of $9 \mu \mathrm{mol} / \mathrm{L}$ (reference 17-41) again with a normal value for glycine $(5 \mu \mathrm{mol} / \mathrm{L}$, reference $2-13)$.

He was treated with oral L-serine supplementation starting in a relatively low dose of 4 times 2 grams/day which was later increased to 4 times 3 grams/day $(140 \mathrm{mg} / \mathrm{kg} /$ day $)$
(Table 1). Significant improvement in general well-being, physical fitness and behaviour were noted within weeks after starting the amino acid therapy and subsequently his absence seizures disappeared. During a three years follow-up his EEG recordings remained free of epileptic activity and ethosuximide medication was successfully discontinued. The values of CSF and plasma serine normalised during treatment. His CSF serine rose from 9 to $29 \mu \mathrm{mol} / \mathrm{L}$ after 4 months of treatment. Developmental testing showed no progress in IQ score (IQ 50).

His sister was 15 years old at the time of diagnosis. She was born after an uneventful pregnancy with a normal head circumference, weight and height. Her early developmental milestones were normal, but developmental delay became evident after four years of age. Similar to her brother, she presented with absence seizures, albeit at an earlier age of five years. Her EEG also showed the typical $3 \mathrm{~Hz}$ bilateral synchronous spike-wave complexes, but with a more frontal predilection. She was treated with ethosuximide and levatiracetam, but despite this had daily absences. She suffered from significant behavioural problems and mood disturbances. In addition to her neurological and behavioural complaints she had severe adiposity and used a wheelchair, because of pain in feet, ankles and knees. The latter complaints were likely caused by generalised hyperlaxity in combination with severe obesity. Prader -Willi syndrome was ruled out by means of molecular testing. Upon formal developmental testing her IQ was shown to be 55 .

Her head circumference was $57 \mathrm{~cm}$ (75th centile), her height was $173 \mathrm{~cm}$ and weight $99 \mathrm{~kg}$ (19 $\mathrm{kg}$ above the 98th centile). Her neurological exam was unremarkable, excluding a neurological cause for her pain symptoms. The latter was further supported by a normal EMG. MRI of the brain showed normal results. She also had striae rubra on lower abdomen and upper legs similar to her brother. Amino acid analysis showed a low plasma serine concentration (56 $\mu \mathrm{mol} / \mathrm{L}$, reference $70-174)$, with a glycine value in the normal range $(175 \mu \mathrm{mol} / \mathrm{L}$, reference $147-321)$. The girl refused to undergo a lumbar puncture to obtain pretreatment CSF amino acid values.

She was treated with 4 times 3 grams L-serine/day $(120 \mathrm{mg} / \mathrm{kg} /$ day) (Table 1). Follow-up revealed a significant improvement in behaviour, ambulance and quality of life. Absences stopped, both anti-epileptic drugs were discontinued and her follow-up EEG was free of epileptic activity. During treatment normal values for plasma and CSF serine were obtained. Repeated developmental testing showed no change in IQ score.

In both children the amino acid therapy was well tolerated and no side effects were reported during the three years follow-up. Enzymatic analysis of 3-PGDH activity in cultured skin fibroblasts displayed a deficient value in both patients (6.8 and 3.1 respectively normal values $33.1 \pm 1.9$ 
Table 1 Comparison of clinical and biochemical features in infantile and juvenile 3-PGDH deficiency

\begin{tabular}{|c|c|c|c|}
\hline & Infantile 3-PGDH deficiency & Juvenile 3-PGDH deficiency & \\
\hline \multirow[t]{2}{*}{ Clinical features } & $\begin{array}{l}\text { Congenital Microcephaly, severe psychomotor retardation, } \\
\text { intractable seizures, irritable and spasticity }\end{array}$ & \multicolumn{2}{|c|}{$\begin{array}{l}\text { Absence seizures, moderate } \\
\text { developmental delay behavioural } \\
\text { abnormalities Joint laxicity and striae }\end{array}$} \\
\hline & $\begin{array}{l}\text { Occasionally megaloblastic anemia cataracts, hypogonadism, } \\
\text { adducted thumbs, inguinal and umbilical hernias }\end{array}$ & & \\
\hline CSF serine $(\mu \mathrm{mol} / \mathrm{L})$ & $6-8$ & $9 *$ & (reference 17-41) \\
\hline CSF glycine $(\mu \mathrm{mol} / \mathrm{L})$ & $1-4$ & $5^{*}$ & (reference 2-13) \\
\hline Plasma serine $(\mu \mathrm{mol} / \mathrm{L})$ & $28-64$ & 63 and 56 & (reference 70-174) \\
\hline Plasma glycine $(\mu \mathrm{mol} / \mathrm{L})$ & $128-190$ & 252 and 175 & (reference 147-321) \\
\hline $\begin{array}{l}\text { Residual enzymatic activity } \\
\text { (nmol/min.mg protein) }\end{array}$ & $3,1-7,3$ & 6,8 and 3,1 & (reference $33,1 \pm 1,9$ ) \\
\hline Treatment & $500-700 \mathrm{mg}$ L-serine $/ \mathrm{kg} /$ day and $200-300 \mathrm{mg}$ glycine $/ \mathrm{kg} /$ day & \multicolumn{2}{|l|}{$100-150 \mathrm{mg} \mathrm{L}$-serine $/ \mathrm{kg} /$ day } \\
\hline Response to treatment & $\begin{array}{l}\text { Control of seizures in some, lowered seizure frequency in all } \\
\text { patients, no progression of psychomotor development }\end{array}$ & \multicolumn{2}{|l|}{$\begin{array}{l}\text { Control of seizures, correction } \\
\text { of behavioural abnormalities }\end{array}$} \\
\hline
\end{tabular}

$\mathrm{CSF}=$ cerebrospinal fluid, $*$ measured only in the boy

(nmol/min.mg protein)), results that were comparable to what was reported in patients with the severe infantile phenotype of 3-PGDH deficiency (Tabatabaie et al. 2009). Mutation analysis of the $P H G D H$ gene confirmed a homozygous c.1129 G>A (p.G377S) mutation in both children. Both parents were shown to be carriers of the mutation confirming autosomal recessive inheritance. The details of the molecular analysis were published elsewhere (Tabatabaie et al. 2009).

\section{Discussion}

This is the first report of a mild form of 3-PGDH deficiency and expands the clinical spectrum of this disorder towards that of a juvenile onset of (absence) seizures and developmental delay. As demonstrated by both children, microcephaly, developmental arrest, spastic tetraplegia and hypomyelination are not necessarily present in 3-PGDH deficiency. In our opinion, L-serine deficiency should be considered in the differential diagnosis of children with developmental delay and seizures.

This family underscores once more the importance of amino acid analysis in the diagnosis of treatable forms of seizures and the need to recognise the significance of low or borderline low values of plasma serine. Because of the influence of dietary amino acids in particular on plasma values, amino acid analysis need to be performed in a fasted state. According to our own (unpublished) data the effects of a single oral load $(100 \mathrm{mg} / \mathrm{kg})$ with either serine or glycine results in a steep rise with a peak concentration between 1-2 hours after loading. Amino acid values return to normal after 4 hours post loading. From this we concluded that in daily practice preferentially amino acids should be analysed after an overnight fast, but otherwise that it would probably be safe to analyse plasma amino acids 4-6 hours after meals. For CSF a single amino acid loading $(100 \mathrm{mg} / \mathrm{kg})$ only marginally influenced CSF serine and values only rose after repeated doses over a period of weeks to months of treatment. Therefore, we consider the influence of meals on CSF serine to be very limited and for this reason remain the preferred diagnostic procedure to confirm serine deficiency.

It was striking that the patients reported here displayed biochemical abnormalities indistinguishable from those with the severe phenotype. Their plasma and CSF serine values were in the same range as what was observed in severely affected infants. The same was true for the 3-PGDH enzyme activity in cultured skin fibroblasts showing a residual activity comparable to that in patients with the infantile phenotype (Tabatabaie et al. 2009). It is obvious that the mild clinical phenotype could not be predicted from the biochemical or molecular analysis and that additional genetic or environmental factors considerably modulate disease severity in serine deficiency. Of particular interest was the fact that these two patients were effectively treated with much lower dosages of L-serine compared to patients with the infantile phenotype. This is remarkable, as we showed earlier that low dosages of L-serine failed to result in clinical and biochemical improvement in patients with the infantile form (de Koning et al. 1998). Therefore, we should revise our former treatment recommendations and add to this that in patients with a mild phenotype a dose of L-serine of $120-150 \mathrm{mg} / \mathrm{kg} /$ day is likely to be sufficient (de Koning 2006). 
In this family both children presented with absence seizures, but it remains to be determined whether this seizure type is specific for this mild form. In the severe infantile phenotype, different clinical seizure patterns and EEG abnormalities were documented, none of which was specific for the disorder or disease progression. Therefore, it seems reasonable to assume that other generalised epilepsies can be present in milder forms of 3-PGDH deficiency. Furthermore, it also needs to be established whether the joint laxity and skin abnormalities are consistent findings of this mild phenotype. These symptoms have not been reported earlier in serine deficiency, but striae rubrae are not very rare in adolescents, which could mean that this is merely a coincidental finding.

\section{Conclusions}

We have shown that the clinical spectrum of phosphoglycerate dehydrogenase deficiency includes a milder form, with juvenile onset of seizures and an excellent response to treatment with L-serine. These patients were biochemically indistinguishable from those with the severe infantile form and the mild clinical phenotype could not be predicted by biochemical or molecular analysis. This family with juvenile 3-PGDH deficiency once again emphasizes the importance of careful interpretation of (low-borderline) amino acid concentrations and early detection of serine deficiency.

Acknowledgments This work was funded by grant WK05.03 from the Wilhelmina Children's Foundation. We express gratitude to the family for their cooperation in this study.
Open Access This article is distributed under the terms of the Creative Commons Attribution Noncommercial License which permits any noncommercial use, distribution, and reproduction in any medium, provided the original author(s) and source are credited.

\section{References}

de Koning TJ, Duran M, Dorland L et al. (1998) Beneficial effects of L-serine and glycine in the management of seizures in 3phosphoglycerate dehydrogenase deficiency. Ann Neurol $44: 261-265$

de Koning TJ, Jaeken J, Pineda M et al. (2000) Hypomyelination and reversible white matter attenuation in 3-phosphoglycerate dehydrogenase deficiency. Neuropediatrics 31:287-292

de Koning TJ, Duran M, Van ML et al. (2002) Congenital microcephaly and seizures due to 3-phosphoglycerate dehydrogenase deficiency: outcome of treatment with amino acids. J Inherit Metab Dis 25:119-125

de Koning TJ (2006) Treatment with amino acids in serine deficiency disorders. J Inherit Metab Dis 29:347-351

Hausler MG, Jaeken J, Monch E et al. (2001) Phenotypic heterogeneity and adverse effects of serine treatment in 3-phosphoglycerate dehydrogenase deficiency: report on two siblings. Neuropediatrics 32:191-195

Jaeken J, Detheux M, Van ML et al. (1996) 3-Phosphoglycerate dehydrogenase deficiency: an inborn error of serine biosynthesis. Arch Dis Child 74:542-545

Pind S, Slominski E, Mauthe J et al. (2002) V490M, a common mutation in 3-phosphoglycerate dehydrogenase deficiency, causes enzyme deficiency by decreasing the yield of mature enzyme. J Biol Chem 277:7136-7143

Pineda M, Vilaseca MA, Artuch R et al. (2000) 3-phosphoglycerate dehydrogenase deficiency in a patient with West syndrome. Dev Med Child Neurol 42:629-633

Tabatabaie L, de Koning TJ, Geboers AJ et al. (2009) Novel mutations in 3-phosphoglycerate dehydrogenase (PHGDH) are distributed throughout the protein and result in altered enzyme kinetics. Hum Mutat 30:749-756 